\title{
A Mechanism for Glucocorticoid Toxicity in the Hippocampus: Increased Neuronal Vulnerability to Metabolic Insults ${ }^{1}$
}

\author{
ROBERT M. SAPOLSKY ${ }^{2}$ \\ Laboratory of Neuroendocrinology, The Rockefeller University, New York, New York 10021
}

\begin{abstract}
Glucocorticoids appear capable of damaging or destroying hippocampal neurons. There is a progressive loss of such neurons with age, and the process can be prevented by adrenalectomy at mid-age or accelerated by prolonged exposure to high circulating titers of glucocorticoids. The present study examines possible mechanisms for this steroid action. Rats were either adrenalectomized, intact, or treated with corticosterone (CORT) sufficient to produce prolonged elevations of titers in the high physiological range. After 1 week, unilateral hippocampal microinfusions were made with either kainic acid (KA) or 3-acetylpyridine (3-AP). Doses of these hippocampal neurotoxins were chosen to produce small-sized lesions. Treatment with CORT exacerbated the extent of damage following neurotoxin infusion, whereas adrenalectomy attenuated the damage. Additional studies eliminated some potential mechanisms for this phenomenon. CORT did not directly alter the intrinsic toxicity of the compounds but, rather, altered the sensitivity of target cells to them. As evidence, no potentiation of damage in CORTtreated animals occurred in KA-sensitive brain regions lacking CORT receptors. Since CORT did not increase the diffusion or binding of $\left[{ }^{3} \mathrm{H}\right] \mathrm{KA}$ in the hippocampus, it appears unlikely that CORT potentiated toxin-induced damage by influencing the specific mechanism of action of any toxin. Finally, the general nature of the CORT potentiation of damage was supported by the markedly different postulated mechanisms of toxicity of KA and 3-AP. We hypothesize that CORT exerts its extensive catabolic effects upon target cells to produce generalized metabolic vulnerability in hippocampal neurons possessing high concentrations of CORT receptors, thereby sensitizing them to varied metabolic insults.
\end{abstract}

Glucocorticoids are secreted by the adrenal cortex in response to stress and have numerous and potently catabolic effects upon metabolism, reproduction, growth, immune function, and the inflammatory response (Baxter, 1976; Munck et al., 1984). These actions can be viewed as crucial for adaptation to acute stress, as they result in more readily utilized sources of energy and in the suppression of unessential anabolic processes (Munck et al., 1984). In

Received July 13, 1984; Revised November 5, 1984; Accepted November 8, 1984

\footnotetext{
1 This work was supported by a predoctoral grant from the National Institute on Aging. I am grateful to Drs. Lewis Krey and Bruce McEwen for advice throughout all phases of this work.

${ }^{2}$ Correspondence should be sent to the author's present address: $\mathrm{c} / \mathrm{O}$ Peptide Biology Laboratory, Salk institute, P. O. Box 85800 , San Diego, CA 92138.
}

contrast, chronic stress or sustained exposure to glucocorticoids can have numerous pathological consequences (Krieger, 1982).

It has become apparent that prolonged exposure to glucocorticoids can also reduce neuron number. Pharmacologic dosages of the steroid were reported to destroy hippocampal neurons (Muhlen and Ockenfels, 1969); the seemingly anomalous anatomical specificity of this effect was somewhat resolved by the subsequent demonstration that the hippocampus contains the highest concentrations in the brain of receptors for these hormones (McEwen et al., 1968). Glucocorticoids also appear to play a role in the loss of hippocampal neurons which normally occurs with age (Landfield et al., 1981a, b; Sapolsky et al., 1984a, 1985); this process can be decelerated by adrenalectomy at mid-age (Landfield et al., 1981a) or accelerated by prolonged exposure to corticosterone titers within the high physiological range (Sapolsky et al., 1985). This loss of neurons might have profound consequences for the aging process. The hippocampus has been implicated in memory formation and retrieval, neuroendocrine regulation and affective state (Scolville and Milner, 1957; McEwen, 1982; Sapolsky et al., 1984b), and hippocampal damage produces functional deficits similar to those frequently scen during aging (Finch and Hayflick, 1977; Bartus et al., 1982; Sapolsky et al., 1984b).

In this paper we have studied the effects of glucocorticoids on neurotoxin-induced damage to the hippocampus, in order to understand the mechanisms by which glucocorticoids can be toxic. We find that the steroids act in a nonspecific manner to incrcase the destructiveness of varied neurotoxins. These findings suggest that glucocorticoids may induce a catabolic state in hippocampal neurons which increases their vulnerability to a broad range of metabolic insults.

\section{Materials and Methods}

Subjects were male Fischer 344 rats, 3 to 5 months of age, given access to food and water ad libitum and kept on a 14-hr/10-hr light-dark cycle (lights on: 6:00 A. M. to 8:00 P. M.). Subjects were either adrenalectomized (ADX), intact (NT), or treated with $5 \mathrm{mg} /$ day (s.C.) of corticosterone in sesame oil (CORT). Such injections produced prolonged elevation of titers equivalent to those produced by a variety of stressors (see Table I in Sapolsky et al. 1985).

Infusion with Kainic acid. Seven days into treatment, subjects were anesthetized with Brevital and infused stereotaxically with varying quantities of the excitotoxin, kainic acid (KA; Sigma Chemical Co., St. Louis, MO). Infusions were made unilaterally into the hippocampus at the coordinate anteroposterior: $+4.1 \mathrm{~mm}$; mediolateral: 2.1 ; dorsoventral: 2.8 (height of lambda suture set equal to bregma). The KA was prepared daily in acidified saline ( $300 \mu \mathrm{g}$ of ascorbic acid/ml of saline) and was injected with a Hamilton syringe in a $1-\mu l$ volume over $1 \mathrm{~min}$, followed by a $1-\mathrm{min}$ wait for the $K A$ to diffuse. In a series of control studies, subjects were infused unilaterally with $\mathrm{KA}$ in either the hypothalamus (anteroposterior: +3.7 ; mediolateral: 0.8 ; dorsoventral: 8.0 ) or the cerebellum (anteroposterior: -3.0 ; mediolateral: 2.0 ; dorsoventral: 3.0 ).

One week after surgery, subjects were perfused with $10 \%$ formalin, and brains were removed and stored in formalin for 5 days. Sections $(32 \mu \mathrm{m})$ were cut and stained with cresyl violet. Damage to the hippocampus was 
assessed at $\times 40$ magnification, using the criteria of Nadler et al. (1978). The area of damage produced by the excitotoxin within a given coronal section and the total volume of damage within a structure were determined with the camera lucida projection technique and AREA/VOLUME program described in the preceding paper (Sapolsky et al., 1985).

Spread of $\left[^{3} \mathrm{H}\right] K \mathrm{~K}$ following infusion. ADX and CORT subjects were microinfused at the hippocampal corrdinates given above with $1 \mu \mathrm{Ci}$ of $\left[{ }^{3} \mathrm{H}\right]$ $\mathrm{KA}$ (Amersham, $5 \mathrm{Ci} / \mathrm{mmol}$ ). This was the molar equivalent to the $0.035 \mu \mathrm{g}$ of unlabeled KA infused in most of the experiments presented below, and the $\left[{ }^{3} \mathrm{H}\right] \mathrm{KA}$ was handled and infused under identical conditions as the unlabeled material. Two hours after surgery, subjects were decapitated and brains were rapidly frozen in dry ice. Sections $(320 \mu \mathrm{m})$ were cut through the entire brain; each section was solubilized in $500 \mu \mathrm{l}$ of $\mathrm{H}_{2} \mathrm{O}$ in a minivial which was filled with $4 \mathrm{ml}$ of Liquiscint (National Diagnostics, Somerville, NJ) and counted. Results are expressed as counts per minute.

Specific binding of KA in the hippocampus. ADX and CORT subjects were decapitated 1 week into treatment. Brains were rapidly removed, frozen, cut at $100-\mu \mathrm{m}$ sections, and mounted. The in vitro binding method of Monaghan and Cotman (1982) for KA receptor autoradiography was modified in order to conduct an in vitro analysis of binding of $\left[{ }^{3} \mathrm{H}\right] \mathrm{KA}$. Slices were preincubated for $10 \mathrm{~min}$ in $50 \mathrm{~mm}$ HEPES-KOH buffer, $\mathrm{pH} 7.1$, on ice, to remove endogenous glutamate. Slices were then divided into six different groups and incubated on ice for $30 \mathrm{~min}$ with three different molar concentrations of $\left[{ }^{3} \mathrm{H}\right] \mathrm{KA}(27,54$, and $70 \mathrm{nM})$, with or without a 1000-fold excess of cold KA. Slices were given two 30-sec rinses in ice-cold buffer to remove free ligand, briefly frozen on dry ice while the surface liquid was aspirated, and dried on a slide-heater. The hippocampus was then scraped off of each slice, solubilized in $500 \mu$ of $\mathrm{H}_{2} \mathrm{O}$, and counted in Liquiscint. Typically, some overlying cortex was included. For this reason, and because $100-\mu \mathrm{m}$ sections were incubated (which almost certainly did not absorb the ligand as readily as the thinner sections in prior studies (Monaghan and Cotman, 1982)), levels of binding differed from previous reports (Monaghan and Cotman, 1982). Aliquots of the solubilized material were removed for protein determinations (Lowry et al., 1951) before the addition of Liquiscint. Specific binding of $\left[{ }^{3} \mathrm{H}\right] \mathrm{KA}$ was then determined and expressed as femtomoles of $\left[{ }^{3} \mathrm{H}\right] \mathrm{KA}$ bound per milligram of protein.

Infusion of 3-acetylpyridine. In a second set of experiments, ADX, INT, or CORT subjects were microinfused with $0.15 \mu \mathrm{l}$ of the neurotoxin, 3 acetylpyridine (3-AP, Sigma) at the same hippocampal coordinates. The toxin was dissolved in $0.85 \mu \mathrm{l}$ of acidified saline, and injections, post-surgical treatment of animals, histology, and assessment of hippocampal damage were as described above for $\mathrm{KA}$

Statistics. Statistical comparisons were made either with independent $t$ tests, or one- or two-way analysis of variance (ANOVA) followed by Scheffe tests.

\section{Results}

KA produced damage within the hippocampus in a dose-related manner (Table I). As previously reported (Nadler, 1979; Coyle; 1983), there were differing sensitivities of different hippocampal cell fields to the excitotoxin, with the CA3 coll ficld bcing cxquisitely sensitive, followed by CA4, dentate gyrus, CA1, and then CA2. This was not related to the proximity of infusion, as infusion was made at a site equidistant from some of these differentially sensitive cell fields. In agreement with previous reports (Nadler, 1979; Coyle; 1983), only very high dosages of KA induced damage outside of the hippocampus (data not shown). A dose of $0.035 \mu \mathrm{g}$ of KA/hippocampus was

TABLE 1

Dose-response relationship between the concentration of $K A$ microinfused into the hippocampus and extent of hippocampal damage (expressed in cubic micrometers $\times 10^{5}$ )

For each concentration, $n=3$. No damage was ever detectable in the CA2 or subiculum cell fields. Anirnals were killed 1 week after surgery.

\begin{tabular}{crrrrr}
\hline $\begin{array}{c}\text { Ka } \\
\text { Concentration } \\
(\mu \mathrm{g})\end{array}$ & CA1 & CA3a & CA3b & CA4 & DG \\
\hline 0.5 & 233 & 23 & 304 & 159 & 0 \\
0.1 & 33 & 20 & 195 & 210 & 138 \\
0.06 & 26 & 15 & 186 & 133 & 0 \\
0.03 & 0 & 14 & 57 & 12 & 38 \\
0.02 & 0 & 0 & 46 & 0 & 0 \\
\hline
\end{tabular}

chosen for subsequent studies in order to induce low but reliable amounts of damage.

The presence of CORT in subjects increased the extent of KA induced damage in the hippocampus (Table I, Figure 1). Generally, ADX subjects had the smallest volumes of damage, with INT subjects intermediate and CORT subjects having the most damage. The effect was most dramatic in the CA3b region, where CORT subjects had more than a doubling of damage, compared to ADX rats. Trends toward significantly increased volumes of damage were observed in the dentate gyrus, CA4, and CA3a regions, whereas no change occurred in CA1. No damage was detected in CA2 in any subjects. In areas showing increased damage, such as CA3b, the increased volume of damage in CORT subjects was attributable both to greater damage within any given coronal section and to a greater posterior spread of damage. The percentage of subjects in each group showing measurable damage did not differ $\left(\chi^{2}\right.$ analysis, data not shown) and ranged from approximately $50 \%$ of subjects with damage in CA1, CA4, and dentate gyrus to $100 \%$ with CA3 damage.

We then determined whether the increased toxicity of KA in CORT subjects could be attributable to greater presentation of excitotoxin to target cells. This was not the case. CORT administration failed to increase the diffusion of $\left.{ }^{3} \mathrm{H}\right] \mathrm{KA}$ in the brain following hippocampal infusion (Table $|i|)$. The tracer did not spread further anteriorly or posteriorly in CORT subjects than in ADX subjects, and there were no greater amounts of tracer within coronal sections of their hippocampi. In fact, a trend toward greater diffusion of the tracer was noted in ADX subjects. In addition, CORT administration failed to increase the specific binding of $K A$ in the hippocampus (Table IV).

The potentiation of the neurotoxic effects of KA by CORT was limited to the hippocampus. Infusion into the cerebellum caused equal volumes of damage in ADX and CORT subjects $\left(57 \pm 25 \mu \mathrm{m}^{3}\right.$ of damage versus $33 \pm 17$, respectively; $t=0.72$, not significant), whereas infusion into the hypothalamus failed to cause measurable damage in any subjects.

Finally, similar studies were conducted in which ADX, INT, and CORT subjects were infused with 3-AP at the same hippocampal coordinates. At this dosage, damage was noted only in the dentate gyrus, predominantly in the dorsal blade of the region, and the extent of such damage was again potentiated by the presence of corticosterone. ADX subjects sustained $11 \pm 6 \mu \mathrm{m}^{3}$ of damage (3 of 6 subjects showing measurable damage); INT subjects had $40 \pm 33$ $\mu \mathrm{m}^{3}$ (3 of 6 subjects), while CORT subjects had $182 \pm 32 \mu \mathrm{m}^{3}$ (10 of 10 subjects). One-way ANOVA followed by Scheffe tests indicated that CORT subjects had significantly greater volumes of damage than both ADX subjects $(F=17.8, p<0.01)$ and INT subjects $(F$ $=9.12, p<0.05$ ). The potentiation of damage occurred in both the

TABLE ॥

Effects of corticosterone treatment on KA-induced hippocampal damage (expressed in cubic micrometers $\times 10^{5}$ )

Subjects were either adrenalectomized for 1 week before surgery, left intact, or injected subcutaneously daily with $5 \mathrm{mg}$ of corticosterone in sesame oil. Treatments were continued for 1 week after surgery. Subjects were surgically infused with $0.035 \mu \mathrm{g}$ of $\mathrm{KA}$ unilaterally into the hippocampus as coordinates indicated under "Materials and Methods."

\begin{tabular}{lccc}
\hline \multicolumn{1}{c}{ Cell Field } & $\begin{array}{c}\text { ADX } \\
(n=7)\end{array}$ & $\begin{array}{c}\text { INT } \\
(n=7)\end{array}$ & $\begin{array}{c}\text { CORT } \\
(n=15)\end{array}$ \\
\hline CA1 & $8 \pm 5$ & $4 \pm 2$ & $8 \pm 3$ \\
CA2 & 0 & 0 & 0 \\
CA3a & $22 \pm 2$ & $30 \pm 8$ & $32 \pm 6$ \\
CA3b & $55 \pm 10$ & $107 \pm 24$ & $119 \pm 11^{a}$ \\
CA4 & $6 \pm 3$ & $18 \pm 11$ & $14 \pm 5$ \\
DG & $31 \pm 18$ & $40 \pm 27$ & $54 \pm 18$ \\
Total damage & $122 \pm 31$ & $199 \pm 34$ & $227 \pm 43$ \\
\hline
\end{tabular}

a Significant at the 0.05 level, Sheffe test following one-way analysis of variance (as compared with $\mathrm{ADX}$ subjects). Values are mean $\pm \mathrm{SE}$. 

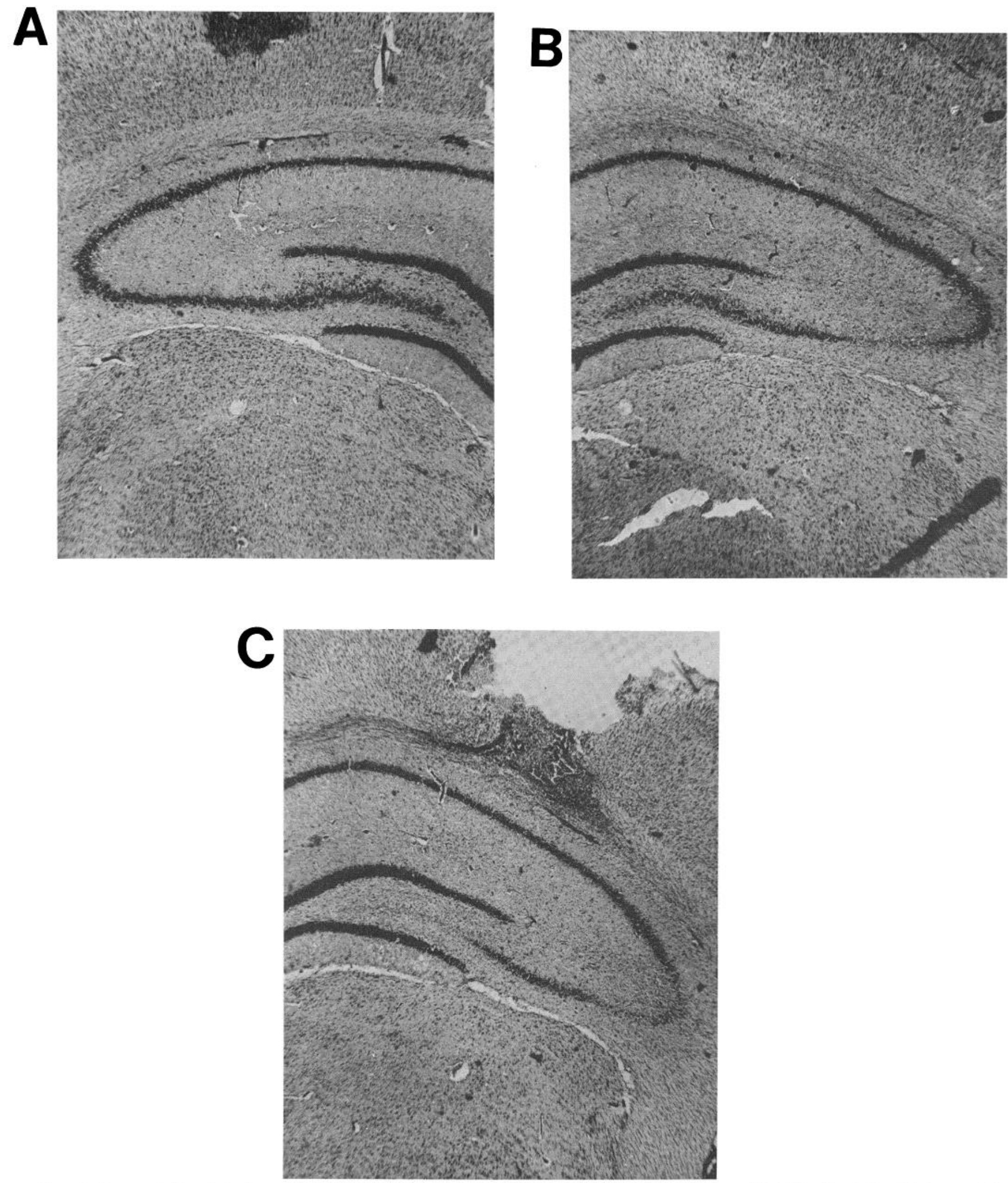

Figure 1. Representative photomicrograph of KA-infused hippocampus of $(A)$ control subject without damage, $(B)$ ADX subject with mild damage in the CA3b region, and $(C)$ CORT-treated subject with damage in CA3a, CA3b, and CA4. $\times 16$ power. 
TABLE III

Extent of diffusion of $\left[{ }^{3} \mathrm{H}\right] K A$ throughout the brain following microinfusion into the hippocampus

Diffusion is expressed in thousands of $\mathrm{cpm}$ per $300-\mu \mathrm{m}$ coronal section. For each group, $n=4$. Values are mean $\pm \mathrm{SE}$. Anterior-posterior coordinate is expressed in micrometers anterior of lambda suture. ADX subjects showed a nonsignificant trend toward greater diffusion of the tracer (two-way analysis of variance; treatment $\times$ anterior-posterior coordinate, $p<0.10$ ).

\begin{tabular}{lcc}
\hline \multicolumn{1}{c}{ Anterior-Posterior Coordinate } & $\begin{array}{c}\text { CORT } \\
\text { Treated }\end{array}$ & $\begin{array}{c}\text { ADX } \\
\text { Subjects }\end{array}$ \\
\hline 1200 ; end of hippocampus & $0.6 \pm 0.1$ & $0.2 \pm 0.1$ \\
1500 & $0.8 \pm 0.1$ & $0.4 \pm 0.2$ \\
1800 & $0.8 \pm 0.1$ & $0.7 \pm 0.3$ \\
2100 & $1.3 \pm 0.1$ & $0.9 \pm 0.4$ \\
2400 & $1.5 \pm 0.1$ & $0.9 \pm 0.3$ \\
$2700 ;$ dorsal and ventral hippo & $1.5 \pm 0.2$ & $2.1 \pm 0.7$ \\
$\quad$ campi merge & & \\
3000 & $2.5 \pm 0.2$ & $2.7 \pm 1.1$ \\
$3300 ;$ most anterior extent of & $2.8 \pm 0.3$ & $3.3 \pm 1.3$ \\
ventral hippocampus & & \\
3600 & $3.0 \pm 0.6$ & $5.0 \pm 1.7$ \\
3900 & $3.4 \pm 1.0$ & $5.5 \pm 1.8$ \\
$4200 ;$ infusion site & $3.5 \pm 1.2$ & $7.5 \pm 2.8$ \\
4500 & $3.3 \pm 0.7$ & $6.2 \pm 1.5$ \\
4800 & $4.5 \pm 0.5$ & $5.9 \pm 0.8$ \\
5100 & $4.0 \pm 1.1$ & $6.0 \pm 1.7$ \\
$5400 ;$ most anterior extent of & $3.4 \pm 1.1$ & $6.1 \pm 1.7$ \\
$\quad$ hippocampus & & \\
5700 & $3.0 \pm 1.0$ & $5.2 \pm 1.8$ \\
6000 & $1.2 \pm 0.4$ & $2.6 \pm 0.8$ \\
6300 & $0.7 \pm 0.2$ & $2.9 \pm 0.5$ \\
$6600 ;$ septum & $0.4 \pm 0.1$ & $1.5 \pm 0.5$ \\
6900 & $0.4 \pm 0.2$ & $1.0 \pm 0.4$ \\
$7200 ;$ anterior commissure & $0.3 \pm 0.1$ & $0.6 \pm 0.1$ \\
7500 & $0.2 \pm 0.1$ & $0.4 \pm 0.1$ \\
7800 & $0.2 \pm 0.1$ & $0.4 \pm 0.1$ \\
8100 & $0.2 \pm 0.1$ & $0.2 \pm 0.1$ \\
8400 & $0.1 \pm 0.1$ & $0.2 \pm 0.1$ \\
8700 & $0.1 \pm 0.1$ & $0.3 \pm 0.1$ \\
9000 & $0.1 \pm 0.1$ & $0.3 \pm 0.1$ \\
\hline & & \\
& & \\
& &
\end{tabular}

TABLE IV

Binding of $\left[{ }^{3} \mathrm{H}\right] \mathrm{KA}$ by $100-\mu \mathrm{m}$ hippocampal slices from adrenalectomized and corticosterone-treated subjects ${ }^{a}$

\begin{tabular}{|c|c|c|c|}
\hline & \multicolumn{3}{|c|}{ Concentration of $\left[{ }^{3} \mathrm{H}\right] \mathrm{KA}(\mathrm{nM})$} \\
\hline & 27 & 54 & 70 \\
\hline & \multicolumn{3}{|c|}{ fmol/mg of protein } \\
\hline $\operatorname{ADX}(n=5)$ & $134 \pm 73$ & $212 \pm 64$ & $502 \pm 191$ \\
\hline $\operatorname{CORT}(n=5)$ & $86 \pm 19$ & $106 \pm 28$ & $325 \pm 48$ \\
\hline
\end{tabular}

a $A D X$ and CORT subjects did not differ significantly in binding (two-way analysis of variance)

dorsal and ventral blades of the dentate gyrus. INT and ADX subjects did not statistically differ from each other, and the percentage of subjects showing damage did not differ ( $\chi^{2}$ analysis).

\section{Discussion}

Prolonged exposure to elevated titers of glucocorticoids can be toxic to populations of hippocampal neurons (Muhlen and Ockenfels, 1969; Landfield et al., 1981a; Sapolsky et al., 1985). As outlined in the introduction, this effect appears to have physiologic ramifications accounting for at least some of the loss of neurons in the hippocampus with age. Because of the evidence implicating hippocampal neuronal damage or loss in a number of cognitive, neuroendocrine and affective dysfunctions which are frequent concomitants of aging, we considered it important to understand the mechanisms by which glucocorticoids can be toxic to hippocampal neurons. The present study provides evidence that glucocorticoids act in a nonspecific manner to increase neurotoxin-induced damage in the hippocampus.

Two different neurotoxins were more destructive to neurons within specific hippocampal cell fields in subjects exposed to prolonged and elevated titers of CORT. In both cases, the region of the hippocampus most vulnerable to the neurotoxin was also most vulnerable to the interaction between CORT and the neurotoxin. In the case of KA, the effect was most marked in the CA3b cell field, with trends toward increased damage in most other areas of the structure. The dentate gyrus, in contrast, was most sensitive to both 3-AP and to 3-AP coupled with CORT. The potentiation of the action of both toxins was due to greater damage per subject, rather than more CORT-treated subjects being above threshold for detectable damage. With both toxins, greater spread of damage and more intense damage at the infusion site were noted. Generally, volumes of damage in INT subjects were intermediate between those of CORT and ADX subjects.

The potentiation of the efficacy of these toxins by corticosterone could be attributable to a number of mechanisms, and the present data allow some to be eliminated. First, glucocorticoids could act upon the toxin molecule itself, in some manner increasing its intrinsic toxicity. Were this rather unlikely possibility occurring, KA-induced damage should have been greater in CORT than in ADX rats in any part of the brain normally sensitive to the toxin. This was not observed. No potentiation of KA-induced damage by CORT treatment occurred in the cerebellum, a region with ample KA receptors (Monaghan and Cotman, 1982) but lacking appreciable numbers of corticosterone receptors (McEwen, 1982). Thus, the KA molecule was not itself intrinsically more toxic in these subjects. Furthermore no measurable damage occurred in any subjects in the hypothalamus, an area with ample corticosterone receptors (McEwen, 1982) but lacking appreciable concentrations of KA receptors (Monaghan and Cotman, 1982). Thus, glucocorticoids did not induce KA to act in a novel, non-receptor-mediated manner. Instead, the potentiation appeared to occur only in cell regions, such as CA3, CA4, and dentate gyrus of the hippocampus, possessing appreciable amounts of receptors for both glucocorticoids and KA (McEwen, 1982; Monaghan and Cotman, 1982).

Thus, it appears that glucocorticoids acted upon target cells to increase their sensitivity to the toxins. This could be through some mechanism specific to that particular toxin, increasing its diffusion, absorption, or retention in target cells. This possibility is made unlikely for numerous reasons. First, the tracer study in Table III indicated that CORT treatment failed to increase the diffusion or retention of $\left[{ }^{3} \mathrm{H}\right] \mathrm{KA}$ in the hippocampus. Second, CORT did not increase the numbers of specific receptors for KA in the hippocampus. Finally, if CORT acted upon CORT-sensitive neurons to increase their binding or retention of KA, treatment of KA-infused rats with CORT should have produced results equivalent to increasing the dosage of KA infused into CORT-free rats. This was not the case, as CORT subjects in Table II did not merely resemble ADX subjects given a greater dose of KA. In the dose-response data of Table I, all areas of the hippocampus (with the possible exception of dentate gyrus) showed equal sensitivities to increasing dosages of KA. In contrast, CORT rats showed a marked increase in damage only in CA3b, with trends in CA4 and dentate gyrus.

Thus, we do not believe that glucocorticoids act to increase the intrinsic toxicity of any given toxin or to increase the sensitivity of hippocampal neurons to the specific mechanism of action of any toxir. Instead, we speculate that glucocorticoids act in a nonspecific manner to induce a catabolically vulnerable state in neurons such that numerous insults, of varied etiology and pathogenesis, are more lethal to the cells. Glucocorticoids have catabolic effects in target tissues throughout the body, including inhibition of glucose uptake, glycogen synthesis, cell replication, and protein synthesis (Baxter, 1976; Munck et al., 1984). Although the metabolic actions of glu- 
cocorticoids are not as well understood in the brain as in, for example, fat or muscle cells, the steroids appear to have some similar catabolic effects in neurons, especially in developing brains (Balazs and Cotterrell, 1972; Gumbinas et al., 1973; Howard and Benjamins, 1975; Landgraf et al., 1978; DeKosky et al., 1984).

This concept of glucocorticoid action is further supported by the differing mechanisms of KA and 3-AP neurotoxicity in the hippocampus. Although the precise neurotoxic actions of KA are disputed (Garthwaite and Garthwaite, 1983), they most likely involve a neuromodulatory release of hippocampal glutamate, a structural analogue of $K A$ and a putative neurotransmitter with demonstrated excitotoxic potential (Ferkany et al., 1982; Coyle, 1983). In contrast, 3-AP is a competitive antagonist of nicotinic acid (Hicks, 1955; Coggeshall and MacLean, 1958; Desclin and Escubi, 1974). In addition, the two toxins differ in which hippocampal neurons are most vulnerable to their effects, and in both cases, it is the most vulnerable cell fields which are made even more so by the presence of glucocorticoids. Finally, as further evidence of the heterogeneous metabolic insults whose potencies are increased by prolonged corticosterone exposure, we have obtained preliminary evidence that hypoxic-ischemic injury to the hippocampus is most pronounced in CORT subjects. This effect is limited to the CA1 cell field ( $R$. Sapolsky and W. Pulsinelli, manuscript in preparation).

in conclusion, the present study suggests that glucocorticoids act to increase, in some manner, the vulnerability of hippocampal neurons to a variety of metabolic insults. Whether the steroids are also capable of ultimately killing the neurons without additional metabolic challenges cannot be determined from the present data. Wo arc currently examining whether neurons are more sensitized by the presence of glucocorticoids before or after the insult, some of the biochemical events which might mediate this phenomenon, and the specific role of such sensitization in hippocampal neuron loss during aging.

\section{References}

Balazs, R., and M. Cotterrell (1972) Effect of hormonal state on cell number and functional maturation of the brain. Nature (Lond.) 236: 348-350.

Barlus, R., R. Dean, B. Beer, and A. Lippa (1982) The cholinergic hypothesis of geriatric memory dysfunction. Science 217: 408-417.

Baxter, J. (1976) Glucocorticoid hormone action. In Pharmacological Therapy, F. Gill, ed., Vol. 2, pp. 605-635, Pergamon Press, Oxford.

Coggeshall, R., and P. MacLean (1958) Hippocampal lesions following administration of 3-acetylpyridine. Proc. Soc. Exp. Biol. Med. 98: 687-688.

Coyle, J. (1983) Neurotoxic action of kainic acid. J. Neurochem. 41: 1-11.

Desclin, J., and J. Escubi (1974) Effects of 3-acetylpyridine on the central nervous system of the rat, as demonstrated by silver methods. Brain Res. 77: 349-364.

DeKosky, S., S. Scheff, and C. Cotman (1984) Elevated corticosterone levels: A possible cause of reduced axon sprouting in aged animals. Neuroendocrinology 38: 33-38.

Ferkany, J., R. Zaczek, and J. Coyle (1982) Kainic acid stimulates excitatory amino acid neurotransmitter release at presynaptic receptors. Nature (Lond.) 298: 757-759.

Finch C., and L. Hayflick (1977) Handbook of the Biology of Aging, Van Nostrand Reinhold Co., New York.

Garthwaite J., and G. Garthwaite (1983) The mechanism of kainic acid neurotoxicity. Nature (Lond.) 305: 129-131.

Gumbinas, M., M. Oda, and P. Huttenlocher (1973) The effects of corticosteroids on myelination of the developing rat brain. Biol. Neonate 22: 355366.

Hicks, S. (1955) Pathologic effects of antimetabolites. I. Acute lesions in hypothalamus, peripheral ganglia and adrenal medulla caused by 3acetylpyridine and prevented by nicotinamide. Am. J. Pathol. 31: 189197.

Howard, E., and J. Benjamins (1975) DNA, ganglioside and sulfatide in brains of rats given corticosterone in infancy, with an estimate of cell loss during development. Brain Res. 92: 73-87.

Krieger, D. T. (1982) Cushing's syndrome. Monogr. Endocrinol. 22: 142.

Landfield, P., R. Baskin, and T. Pitler (1981a) Brain aging correlates: Retardation by hormonal-pharmacological treatments. Science 214: 581-584.

Landfield, P., L. Braun, T. Pitler, J. Lindsey, and G. Lynch (1981b) Hippocampal aging in rats: A morphometric study of multiple variables in semithin sections. Neurobiol. Aging 2: 265-275.

Landgraf, R., A. Mitro, and J. Hess (1978) Regional net uptake of 14Cglucose by rat brain under the influence of corticosterone. Endocrinol. Exp. 12: 119-128.

Lowry, O. H., N. J. Rosebrough, A. L. Farr, and R. J. Randall (1951) Protein measurement with the Folin phenol reagent. J. Biol. Cherrl. 193: 265-275.

McEwen, B. (1982) Glucocorticoids and hippocampus: Receptors in search of a function. In Current topics in Neuroendocrinology P. Ganten and D. Pfaff, eds., Vol. 2, pp. 23-47, Springer-Verlag, Berlin.

McEwen, B., J. Weiss, and L. Schwartz (1968) Selective retention of corticosterone by limbic structures in rat brain. Nature (Lond.) 220: 911-912.

Monoghan, D., and C. Cotman (1982) The distribution of [3H]kainic acid binding sites in rat CNS as determined by autoradiography. Brain Res. 252: $91-100$

Muhlen, K., and H. Ockenfels (1969) Morphologische Veranderungen im Diencephalon und Telencephalon nach Sotrungen des Regelkreises Adenohypophyse-Nebennierenrinde. III. Ergebnisse bein Meerschweinchen nach Verabreichung von cortison und Hydrocortison. Z Zellforsch. 93: 126-141.

Munck, A., P Guyre, and N. Holbrook (1984) Physiological functions of glucocorticoids in stress and thier relation to pharmacological actions. Endocr. Rev. 5: 25-44.

Nadler, J. (1979) Kainic acid: Neurophysiological and neurotoxic actions. Life Sci. 24: 289-300.

Nadler, J., B. Perry, and C. Cotman (1978) Intraventricular kainic acid preferentially destroys hippocampal pyramidal cells. Nature 271: 676-678.

Sapolsky, R. M., L. C. Krey, B. S. McEwen, and T. C. Rainbow (1984a) Do vasopressin-related peptides induce hippocampal corticosterone receptors? Implications for aging. J. Neurosci. 4: 1479-1485.

Sapolsky, R., L. Krey, and B. McEwen (1984b) Glucocorticoid-sensitive hippocampal neurons are involved in terminating the adrenocortical stress. response. Proc. Natl. Acad. Sci. U. S. A. 81: 6174-6177.

Sapolsky, R. M., L. C. Krey, and B. S. McEwen (1985) Prolonged glucocorticoid exposure reduces hippocampal neuron number: Implications for aging. J. Neurosci. 5: 1221-1226.

Scolville W., B. Milner (1957) Loss of recent memory after bilateral hippocampal lesions. J. Neurol. Psychiatry 20: 11-21. 